\title{
Open Symphony: Creative Participation for Audiences of Live Music Performances
}

\author{
Yongmeng Wu, Leshao Zhang, Nick Bryan-Kinns, Mathieu Barthet Member, IEEE \\ E-mail: m.barthet@qmul.ac.uk
}

\begin{abstract}
Most contemporary Western performing arts practices restrict creative interactions from audiences. Open Symphony is designed to explore audience-performer interaction in live music performance assisted by digital technology. Audiences can conduct improvising performers by voting for various musical 'modes'. Technological components include a web-based mobile application, a visual client displaying generated symbolic scores, and a server service for the exchange of creative data. The interaction model, app and visualisation were designed through an iterative participatory design process. The visualisation communicates audience directions to performers upon which to improvise music, and enables the audience to get feedback on their voting. The system was experienced by about 120 audience and performer participants ( 35 completed surveys) in controlled (lab) and "real world" settings. Feedback on usability and user experience was overall positive and live interactions demonstrate significant levels of audience creative engagement. We identified further design challenges around audience sense of control, learnability and compositional structure.
\end{abstract}

Index Terms - participatory live music performance, music interaction, audience engagement, audience experience, music visualization, stage augmentation, creativity, music improvisation, multimedia, mobile, web, software engineering

\section{INTRODUCTION}

C Ontemporary Western music performances are predominantly presentational, in that performers prepare and provide music for another group, the audience [1]. Audiences have an important role in creating the setting and experience of musical performances [2]. Without, performers may play less expressively as for some musicians 'playing expressively' means 'communicating emotions' to an audience [3]. Audience participation in musical performance can be manifested in a broad range of ways, from a receiver sense (e.g. sitting in silent contemplation of sounds) to a more active form of participation involving actions shaping the performance (e.g. singing along with musicians, common in contemporary pop music concerts), which we coin as creative participation 1 . Western art music has evolved by creating a divide between audiences and performers, from early music forms for the recreation of amateurs to post-Renaissance music for entertainment by highly-skilled professional performers. Certain music traditions developed unwritten rules about audience etiquette constraining the range of behaviors and interactions judged to be acceptable; in some concert halls, 'correct' audiences are expected to sit quietly and applaud only in certain places. The musical communication model that prevails in many cultures and traditions operates in a linear and unidirectional way from

- Y. Wu, L. Zhang, N. Bryan-Kinns and M. Barthet are with the School of Electronic Engineering and Computer Science, Centre for Digital Music, Queen Mary University of London, London E14NS, UK.

Manuscript received 11 February 2016; Accepted 11 November 2016; Published in IEEE Multimedia Magazine January-March 2017 issue.

1. This description of creative participation does not refer here to the semiotics sense implying that the meaning of art works is completed by the receiver, which can be considered as a cognitive form of creative participation (see works of Umberto Eco and Ernst Gombrich mentioned in [4]). composer to performer to the audience-receiver. What if this were revisited - not to replace - but to create alternative musical experiences changing the relationships between the composition, the composer, performers and audiences? Reconfiguring the links between the triad of the artwork, artists and audiences' is one of the remits of participatory art (see Section 2) and is explored within the interactive and performance arts [4]. A remarkable initiative to enrich concert experiences using digital multimedia and internet technology is described in [5]. However such initiative in the field of music interaction does not address the creative participation of audiences during live performance, which is the object of the present and previous works (see e.g. [6] [7] [8] [9]).

In this paper, we present Open Symphony (OS), a new participatory music performance system which explores the creativity and spontaneity of reactive interactions through an ensemble of performers and an audience using mobile technology and data visualisation. The system aims to extend the nature of live music performance by enabling audience members to collaborate in the development of a musical piece - audience members actively influence the music being played in a live environment, creating a mutual experience between multiple audience members and performers. Our novel contributions include a classification taxonomy for participatory live music performance (PLMP) systems (Section 2), a system for audience-performer live musical co-composition based on vote and stage augmentation techniques (Sections 3 and 4 , and an evaluation methodology which may serve as a basis for the assessment of other PLMP systems (Section 5). We conducted two evaluation sessions involving a total of about 120 interacting participants across 14 different performances in two different settings, one controlled, in the lab, and another 
in the wild (14 participants per performance on average, 35 completed surveys). Audience usability results show that the system is overall intuitive but complete learning requires an introduction and a few trials, especially for non-musicians. Thematic analyses of self reports and statistical analyses of live interactions demonstrate that for some participants the system successfully supports creative interactions with performers and fosters engagement in the performance through the sense of agency and being cognitively active, the aesthetics of the stage augmentation, and the uniqueness of the performances contributing to positive affective responses. For others, the system can lead to frustration due to the lack of control while not engaging with the produced experimental music. Such successes and limitations of our system are further discussed in Section 6. Video and photo examples showing live interactions with Open Symphony can be found at the following links: http:/ / bit.ly/os_visvideo and http://bit.ly/os_photos

\section{Audience Participation in the Digital Age}

\subsection{Participatory Music Within Participatory Art}

In contrast to presentational music performance, participatory music performance can be defined as a special type of music practice in which the performer-audience distinctions are blurred and where all participants actively contribute to the musical outcome (based on [1]). Participatory music has a long history in many cultures and took various forms (e.g. early Western madrigals for communal singing, Zimbabwean Mbira music played at ceremonies involving singing, clapping and dancing) which share seed values such as "heightened social interactions" and the inclusion of as many participants as possible whatever their level (ibid.). Motivations for audience participation in modern and contemporary live performing arts (music, theatre, dance, comedy, etc.) include the goal of active spectatorship ("as physical engagement may strengthen mental engagement"), creating communities in which participants are equal by improving the sense of closeness between the audience and performers and between audience members themselves, undermining the boundaries of traditional art forms expectations and behaviors, and reaffirming the liveness in performance given the ever-increasing number of live acts based on pre-recorded media [10]. Interesting parallels can be drawn between some of these motivations and the contemporary perception of audience's role which has recently been suggested to have changed from primarily passive to one "co-creating values" by several authors, while audiences are shown to increasingly want to "shape" their own experience [2].

\subsection{Classification Framework for Participatory Live Mu- sic Performance Systems}

We differentiate participatory art forms according to the level of audience creative participation, from partial, where audiences influence content produced by artists to full, where there are no artist-audience distinctions. Until recently, participatory art forms with partial participation made use of low technology for audience interventions, e.g. a dice in Steve Jackson and Ian Livingstone's fantasy roleplay gamebooks "in which you are the hero!" readers use to determine the progression of the narrative, verbalisations in improvisational theatre and stand-up comedy letting the audience give cues to performers, etc. We, as others [4], believe that human-computer interaction (HCI) and communication technologies have much to offer to participatory art forms in that they provide a platform to mediate and transform creative information between agents (whether human or virtual, co-located or remote), and can help overcoming the boundaries that low technologies impose on creative interactions with the potential to scale them up to large audiences. At the intersections between music and HCI (see e.g. |11|), several techniques and interaction models have been proposed to control access points for technology-mediated audience creative participation in the musical context [12] [13]. In Open Symphony the audience acts as a "meta-composer/conductor" by voting for playing modes [14] communicated to performers through live graphic scores. We present below our framework for classifying participatory live music performance systems, using the framework to position Open Symphony as well as the works of others.

- Audience creative participation level: from partial (Open Symphony) to full (no artist-audience distinctions);

- Audience creative participation motivation: e.g. imitative (following), competitive, contributing, directing/conducting (Open Symphony);

- Agency distribution: how creative agency is distributed, from individual level to collective level, such as audience subgroups ( $|6|$ and Open Symphony), or the entire audience;

- Agency mediation: directness of the agency, from indirect (as in Open Symphony, where performers follow audience instructions and create the sounds) to direct without mediation (where the audience creates sounds themselves [8] [9]);

- Agency degree: whether participants act upon the performance (audience adds content to an existing narrative [15]) or co-create the narrative (as in Open Symphony);

- Agency constraints: from restrictions (as in Open Symphony, where the audience chooses between certain options) to complete freedom (where the audience has full control);

- Creative participation modalities: examples include audition (audience generates sounds [8] [9]), vision [16], multimodal audition and vision (as in Open Symphony, where the audience actions influence projected visuals and sounds mediated by performers, similar to other work |7|, or when audience members produce sounds and visuals with their mobile phones [9]);

- Creative participation media: for example, sound/music [7] [8] [9], lights (where the audience can control light effects [17]), 3D virtual content [18], or text messages [15];

- Creative participation affordances: based e.g. on body and movement (such as dancing [19|), hand/arm gestures |9], votes (as in Open Symphony and other works [7] [17]), or linguistic expressions [15];

- User interfaces: e.g. web-based interfaces (such as Open Symphony [20] and other works [21] [7]), tangible interfaces [22], wearable interfaces [19], or mixed reality 
display [18];

- Situation: co-located (as in Open Symphony, where the audience and performers physically share the same space), remote, or both [15];

- Scalability: from small groups [22] to potentially large audiences (as with Open Symphony and other works [19] [21] [7] [20]).

The musical form of Open Symphony is directed improvisation which can be seen to belong to the experimental music genre associated to non-traditional compositional and performance practices. Several other non-traditional conducting techniques non-mediated by technology make use of playing modes and visual cues, for instance the 'Conduction' system from Lawrence D. "Butch" Morris which proposes an exchange between a composer/conductor and performers that provides the immediate possibility of altering the musical attributes of a performance based on a system of signs [23]. However Morris' Conduction is not concerned with creative participation from the audience.

\section{Design Process and Requirements}

We adopted an iterative participatory design methodology motivated by the proposition "that the people whose activity and experiences will ultimately be affected most directly by a design outcome ought to have a substantive say in what that outcome is" (Caroll and Rosson cited in [4]). Active participation of stakeholders and users (composers, performers, audiences) took place in four different forms over a year: regular design sessions with Kate Hayes, a musician and Guildhall School of Music and Drama (GSMD) graduate; focus groups during pilot experiments with performers; focus groups during rehearsals with performers; and interactive performances with both audiences and performers, including evaluations. The core design team comprised Yongmeng $\mathrm{Wu}$, Leshao Zhang, Mathieu Barthet and Kate Hayes.

We initially explored and defined the problem during design sessions with Hayes, who also acted as the project's music director. From the artistic perspective, one of the intents of Open Symphony is to challenge the role of audience and performer, redefining the direction of musical creativity and expertise resulting in spontaneous and collaborative new music compositions (see [14] for more details). The core design team thus established the following main design requirements (DRs) for a PLMP system for an audience at a small ensemble performance:

- facilitate audience creative participation (DR1),

- support performers' musical expression-performers should not feel controlled by the audience and should be able to express their musical expertise and keep some music credit (DR2),

- allow responsive audience-performer interactions (DR3),

- be intuitive and inclusive by suiting musically untrained audience users (DR4),

- foster audience engagement in the performance (DR5),

- support musical co-composition (DR6),

- and be scalable to large audiences (DR7).

We used mockups and prototyping to discuss models varying creative participation affordances, musical system and GUI designs (the description of these early models and their evaluation lie beyond the scope of this paper). The core design team and performers assessed these prototypes against the DRs. The outcome was a first functional system which was trialled with four professional performers during pilot experiments. We used feedback obtained during focus groups with performers after the trial to refine the design (see Section 4.1); a photo of one of the focus group sessions is available here

We then conducted two cycles of evaluations and improvements of the system based on the feedback of audiences and performers obtained after rehearsals and interactive performances. Our evaluation methods, results and modifications are described in Section 5

\section{Open Symphony System}

\subsection{Artistic and Interaction Models}

In order to establish a creative balance between audience and performers the traditional musical score and central conductor are replaced by deconstructed music playing modes [14] and multiple audience conductors. Our and Hayes' creative interaction model let audience members specify collectively the playing modes which can be seen as typological music attributes, and let performers control morphological music attributes within constraints established by the modes. Performers' interpretations of the modes can be based on pre-composed material either provided by the composer or generated during rehearsal, and are shaped according to expressive intent, e.g. through choices of timing and timbre (see e.g. [24]). The choice of modes is orchestrated by a voting system where electors are audience members and 'candidates' consist in the five following playing modes selected by Hayes: 'Drone' (single sustained note), 'Two-note' (two notes played alternatively), 'Motif' (small set of notes forming a melody), 'Improvisation' (free improvisation), and 'Silence' (no playing). The primordial 'Silence' mode was added based on the results of a focus group with performers. We assumed that the modes would be straightforward concepts for audience to learn and understand, and sufficiently loose constraints leaving performers enough freedom for expression exploiting their expertise and creativity. For such interaction to take place dynamically during a performance, relevant information needs to be presented in a timely and efficient manner. We developed information visualisation techniques to simplify the exchange of creative data, to serve as a cognitive tool to support decision making [25], and for aesthetic purposes. The audience is divided into different groups which are each assigned to a performer automatically upon connection to the user client (see Section 4.3). Group votes are used to generate individual symbolic scores for each performer. The voting system relies on the plurality principle meaning that the winning mode is that with most votes. Mode indications are directive for performers, however the artistic brief leaves them a flexibility in the transitions from one to another (namely to avoid abrupt interruption of musical expression).

\subsection{Creative Communication System Architecture}

The Open Symphony creative communication system consists of three components: i) an audience user client for audience voting; ii) a visual client for presenting visual 
feedback to both audience and performers; iii) a server component supporting the interaction between the visual client and mobile applications.

\subsection{Audience User Client}

The Open Symphony audience user client is a crossplatform and smartphone-friendly web-based application (app) developed in HTML5. This is advantageous as users don't have to download an app prior to interacting. Following visual perception principles |25| we used different visual channels to display different types of data, namely shapes (e.g. circles, triangle, etc.) were associated to performers, and pattern, color, and motion were associated to playing modes. The graphical user interface (GUI) of the app (Figure 1a) displays a shape identifying the assigned performer, a bar chart representing the number of votes for each playing mode in a given group, and buttons to select the playing modes. The vote buttons are located at the bottom of the mobile device which was found to be the best touching area for single hand operation on mobile phones [26]. We chose a dark background color for the GUI to reduce visual disturbance whilst the app is used at a concert setting as would happen with a brightly lit screen. Help instructions can also be accessed by users at any time simply by pressing on a help button.

\subsection{Visual Client}

The visual client generates a graphical score for performers and audience feedback (Figures $1 \mathrm{~b}$ and $1 \mathrm{c}$. The designed score notations build on features of traditional music where time-based events are laid out from left to right as well as on contemporary graphic notations [27]. Each performer has a score timeline starting with the shape they have been assigned to and displaying graphical symbols representing the playing modes. The status of the audience votes is also represented on the score. With this one-to-one mapping, the visual client provides direct feedback on the audience's interaction with the aim of encouraging users to actively participate. Audience votes are sampled at a frequency set up to limit network cluttering (every two seconds in the experiment presented in Section 5. When a new mode is voted for, its symbol appears gradually before reaching its final shape in order to help users follow time and add dynamism to the interface for increased engagement.

\subsection{Server}

The server component which is powered by Node.js, a crossplatform server-side JavaScript framework, is described into details in |20|. Node.js suits the high concurrency, low CPU consumption requirements of Open Symphony. The server caches the instructions from the mobile applications and the visualisation client in memory, allowing to fetch them with representational state transfer (RESTful) application programming interfaces (APIs), thus creating a communication channel for mobile applications and visualisation client. The frequency of requests from visual client to server was set up so that graphical score update occur every $2 \mathrm{~s}$ to avoid too sudden of changes.

\section{Evaluation}

We set out to evaluate audience and performer responses to the novel interaction provided by Open Symphony in two different performance contexts (lab and "in the wild"). Our aim was to gather subjective feedback on the experience through a survey, to explore how the system was actually used, how it changed performance practice, and how it could be improved.

\subsection{Sessions and Participants}

The first session (denoted SA) was hosted in a 'blackbox' performance space at the authors' institution. The performers were four professional musicians (three flutists and bass and alto clarinetist) who were Guildhall School of Music and Drama (GSMD) graduates. Audience participants were recruited through (online) call of participations at QMUL and GSMD. In total 13 participants interacted (including two experimenters, occasionally). The survey was completed by the four performers and 11 recruited audience participants (five males and six females) who belonged to the following age groups: 20-29 (5), 30-39 (4), 40-49 (1), 50+ (1). Audience participants included students (MSc and $\mathrm{PhD}$ ), researchers, a photographer, a teacher and musicians.

The second session (denoted SB) was held as part of the Conference on Human Factors in Computing Systems (CHI 2016) Interactivity track [14] in a large open booth located in the conference's exhibition hall (see Figure 2). The performers were four professional musicians (one violist, two violinists, and bass and alto clarinetist) who were University of California Berkeley (UCB) music graduates. In total 102 different audience participants took part in interactive performances over two consecutive days (including two experimenters, occasionally). The survey was completed by 20 of them (16 males, two females, two N/A) who belonged to the following age groups: 18-24 (1), 25-34 (10), 35-44 (5), 40-49 (1), 50+ (1). Audience participants included researchers, students (PhDs), designers, software developers and lawyers. Two out of the four performers provided feedback in SB.

As the votes from the two experimenters who actively took part in the interactive performances might have influenced other participants' votes, they were included in the log-based behavioral analyses presented in Section 5.4.2 (process similar than in [15]). However, we do not believe that these votes had a major impact on the overall audience participation.

\subsection{Procedure}

In each session the procedure included four main stages: (i) performer rehearsal, (ii) system presentation, (iii) series of interactive performances, and (iv) post-performance survey. During the rehearsal performers were introduced to the system and practiced with the music director first without the technology (system of paper cards and hand signs for mode indications) and then with the technology $(\sim 2-3 \mathrm{H})$. In SA, all participants took part in four pieces where the number 


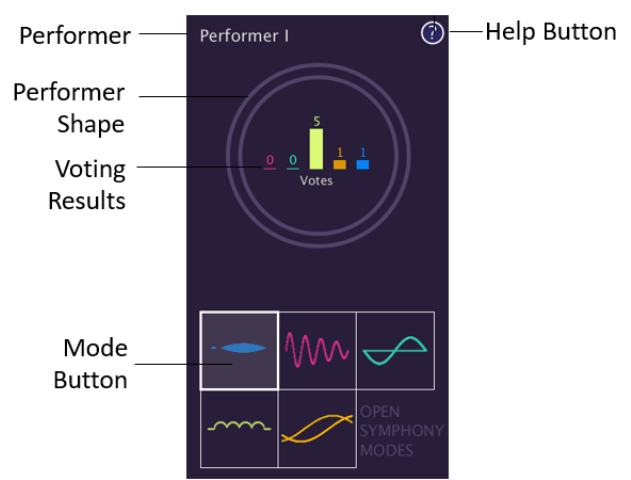

(a) GUI from the Open Symphony app

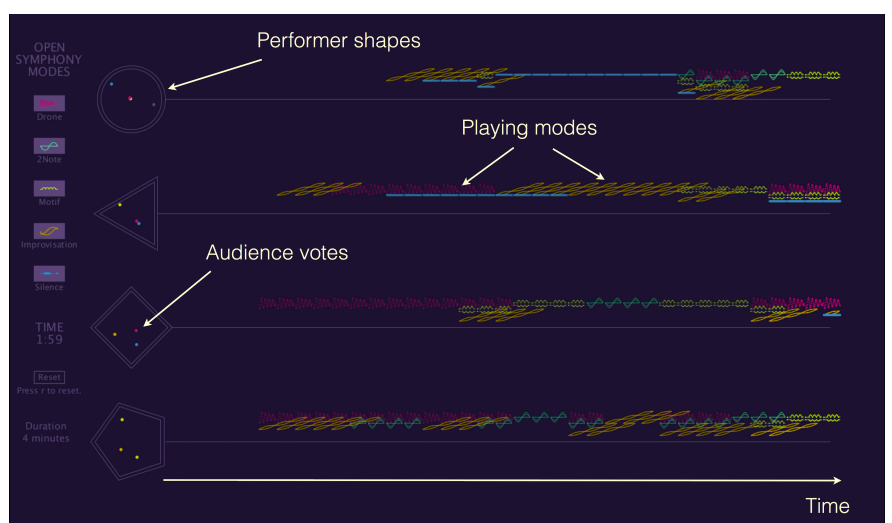

(b) Graphical score (Session A)

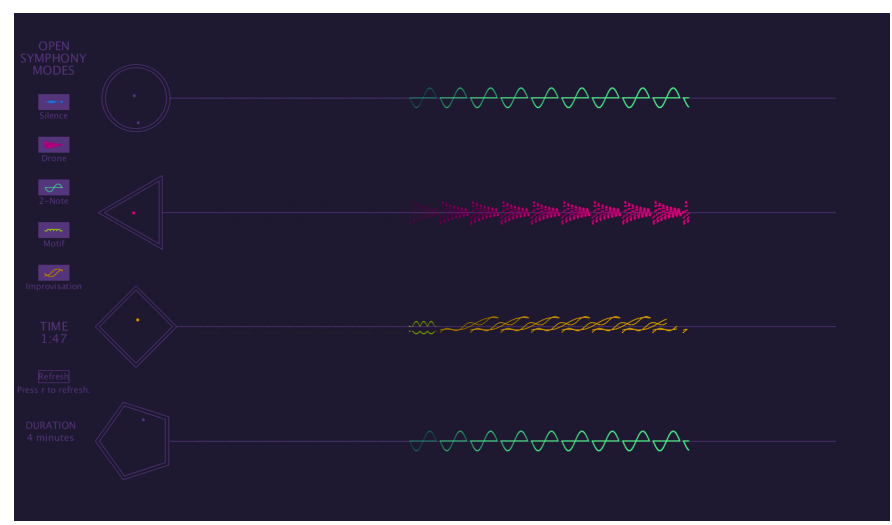

(c) Simplified graphical score (Session B)

Fig. 1. Open Symphony user interfaces: app $1 a$ and graphical score $1 \mathrm{~b}$ and $1 \mathrm{c}$. Graphical scores: from left to right, status bar indicating which modes are enabled and the current time, shapes associated to each performer (audience vote overview appears inside the shapes with colored dots corresponding to votes for specific modes), mode symbols appearing on performer timeline to indicate which mode to play. Mode symbols disappear as time goes by to put forward most recent modes.

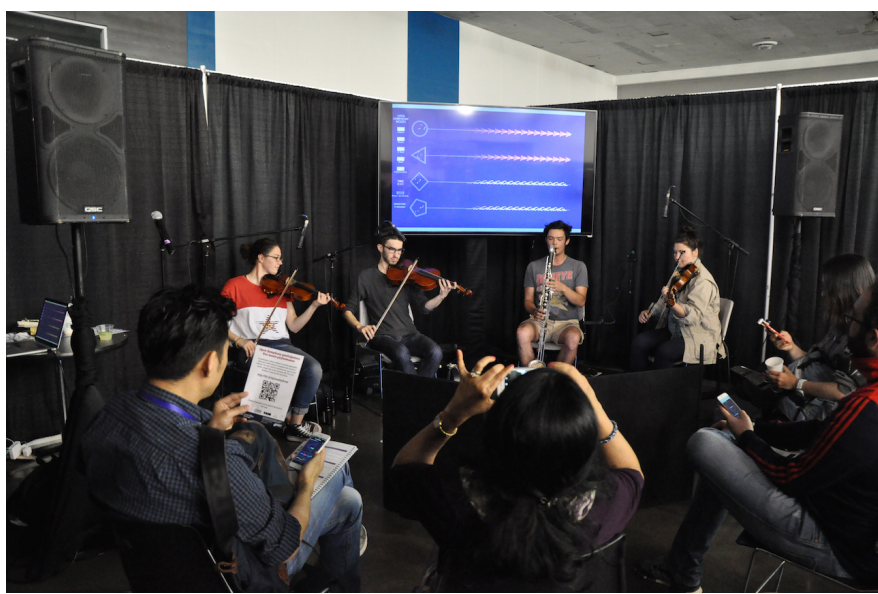

(a)

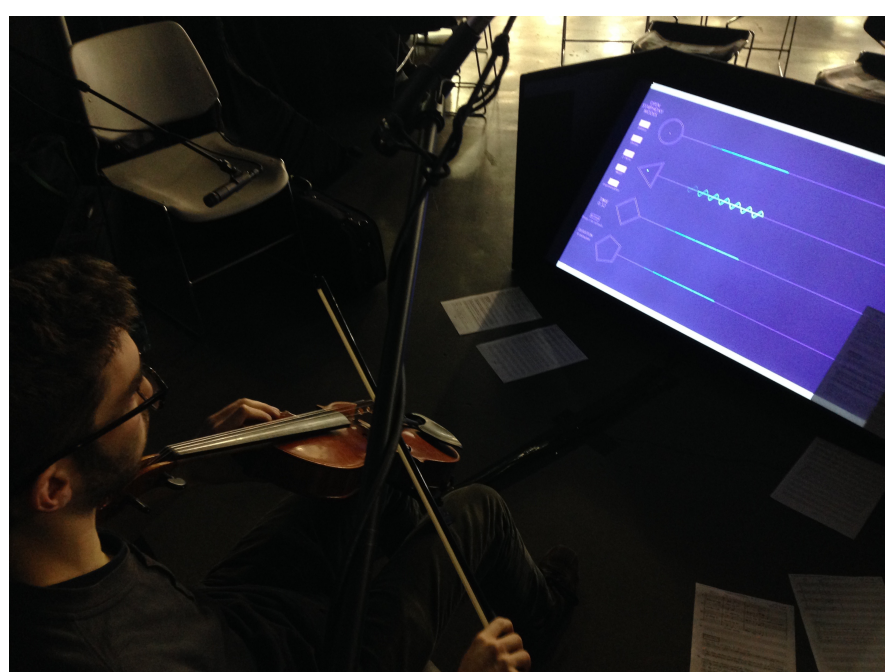

(b)

Fig. 2. Ensemble and first row of audience participants $2 \mathrm{a}$ and performer screen $2 \mathrm{~b}$ for session B. Rich diversity of audience behaviors can be in seen in Figure 2a from left to right, participant reading one of the instruction flyers they had access to, participant taking a photo of the performance, participant looking in the direction of the performers and the main visual display, participant using her mobile phone to interact. The computer generating live graphical scores is located on the left side of the stage.

of active modes was gradually increased (two, three, four and five modes for pieces one to four, respectively) so that audience members could learn the concepts of modes and their influence progressively. The audience was seated. The 
performance and survey lasted about two hours in total. SB comprised ten pieces held on consecutive days mostly with different participants (average of 1.4 performances per participant). Audience members were seated (first rows) and standing at the back. In both sessions, the duration of each piece was fixed to four minutes. During the presentation we introduced the interaction principle and how to connect to (URL or QR code) and use the app. However in SB, not all participants followed the presentation as attendees could join or leave the booth whenever they wanted. Instructions cards (A5 flyers) were at disposal on the booth chairs and tables and distributed to some of the new comers. After two iterations with only two and three modes enabled we decided to keep five modes available for all the other performances as the music was more varied. In both sessions audio/video recordings were made and interaction data was stored on our server.

\subsection{Apparatus and Setting}

In both sessions audience and performers faced each other as in a traditional performance setting (see Figure $2 \mathrm{a}$ for illustration). Two screens connected to the same Apple MacBook displayed the visualisation; for the audience: a large 5 x $3 \mathrm{~m}$ rear projection screen (SA) and a $80^{\prime \prime}$ high definition digital television (SB) placed behind the performers, and for the performers: an HDTV located slightly on the side of the stage area (SA) and on the floor in front of the performers at an angle of $\sim 45^{\circ}(\mathrm{SB})$, see Figure $2 \mathrm{~b}$. In both sessions audience participants were invited to use their mobile devices with either the venue WiFi or mobile broadband. In SA three video cameras were used (performers, audience, and back of the audience). In SB one video camera was used at the back of the audience. In SA, a dimmed lighting was used for projection allowing performers to see each other whilst keeping the intimacy sought for in concert situation. In $\mathrm{SB}$, normal lighting was used suiting the open booth configuration in an exhibition hall.

\subsection{Survey}

In both sessions we conducted a survey using online (SA) and paper (SB) self-completion questionnaires for audience (SA, SB) and performer (SA) participants. We collected reflective feedback for SB performers by email for practical reasons. Questionnaires were chosen over interviews to be able to collect feedback for a whole group while participants were still fresh about the performance, and also to prevent the experiment from being too long for attention and availability reasons. In the statistical analyses that follow a Type I error rate of $\alpha=.05$ was chosen.

\subsubsection{Subjective Evaluations}

Participants rated their experience and the system on topics expressing enabling/disabling factors (see Figure 3 using five-point Likert items from 'Strongly disagree' (1) to 'Strongly agree' (5). Our questionnaires included traditional usability metrics to test ease of use, understanding and effectiveness (see Figure $3 a$ topics 1 to 6 , and Figure $3 b$ topics 1 and 2). They also included user experience (UX) metrics to enquiry about perceived satisfaction; the need for audience creative participation and whether creative collaboration within audience members occurred (DR1); user interface implementation choice (mobile phone); the sense of engagement (DR6); the pedagogical and retention potential of the system (see Figure 3a topics 7 to 11 and Figure $3 \mathrm{~b}$ topics 3 to 7). Fewer topics were used in SB due to the shorter amount of time for participants. In order to test the agreement between participants (raters) we computed inter-rater reliability (IRR) measures adapted to ordinal data [28]. The intra-class correlation (ICC) was used for SA which did not present missing data. A strong significant agreement was found, ICC $(\mathrm{A}, 11)=0.764$ [FTest, H0: $\mathrm{r} 0=0$; H1: $\mathrm{r} 0>0$; $\mathrm{F}(10,60.9)=5.58, \mathrm{p}<.0001]$. As results for SB included missing data we assessed IRR using the bootstrapped Krippendorff's Alpha which also showed significant agreement (Alpha $>0)$, Alpha $=0.21$, $95 \%$ CI [.06, .37], although to a lesser extent than in SA. Several limitations are highlighted by the ratings: the score visualisations were not found clear at the significant level (SB), and did not systematically help to follow the music (SA); the app was not found easy to use at the significant level (SB only). The results also highlight several successful aspects of the system: none of the participants significantly disagreed to any of the enabling topics in either session (upper limit of median notch below 3), i.e. in the worst cases participants expressed neutral judgements to enabling topics. The participants were satisfied with their overall experience and enjoyed interacting with the performers at the significant level (SA and SB); participants found the app easy to use and its interface clear (SA) and felt engaged in the performance (SA) at the significant level. The use of mobile device was not found to prevent paying attention to the performance significantly (SA); participants reported to have enjoyed collaborating with audience members and also expressed to be keen to participate again, at the significant level (SA: polar question results, not reported here, and SB).

\subsubsection{Behavioral Analyses}

5.4.2.1 Creative interaction data: We aimed to analyse how frequently participants voted and whether differences occurred between playing modes. We compared vote interactions for pieces with five available modes (i.e. one piece for session A and eight pieces for session B). From the server logs, we computed $\bar{v}_{i j k_{j}}$ the mean vote frequency per participant for mode $i \in[1 ; 5]$, session $j \in[1 ; 2]$ and session piece $k_{j=1}=4$ (one piece) and $k_{j=2} \in[3 ; 10]$ (eight pieces). For a given mode and piece, $\bar{v}$ is obtained by dividing the total number of votes for the mode by the number of participants to the piece and is expressed in number of votes per minute. We conducted a two-way analysis of variance (Type II sum of squares ANOVA) that examined the effects of Session (two levels) and Mode (five levels) on the mean vote frequency $\bar{v}$. Our dependent variable, the mean vote frequency $\bar{v}$, did not depart from a normal distribution for the groups formed by the combination of the levels of Session and Mode as assessed by the Anderson-Darling test (A2 always below critical level). There was homogeneity of variance between groups as assessed by Levene's test for equality of error variances, $F(9,35)=1.30, p=.27$. No interaction effect between Session and Mode was found, $F(4,35)=2.24, p=.08$. No significant main effect of the Session was found, $\mathrm{F}(1,35)=0.59, \mathrm{p}>.45$, indicating that the mean 


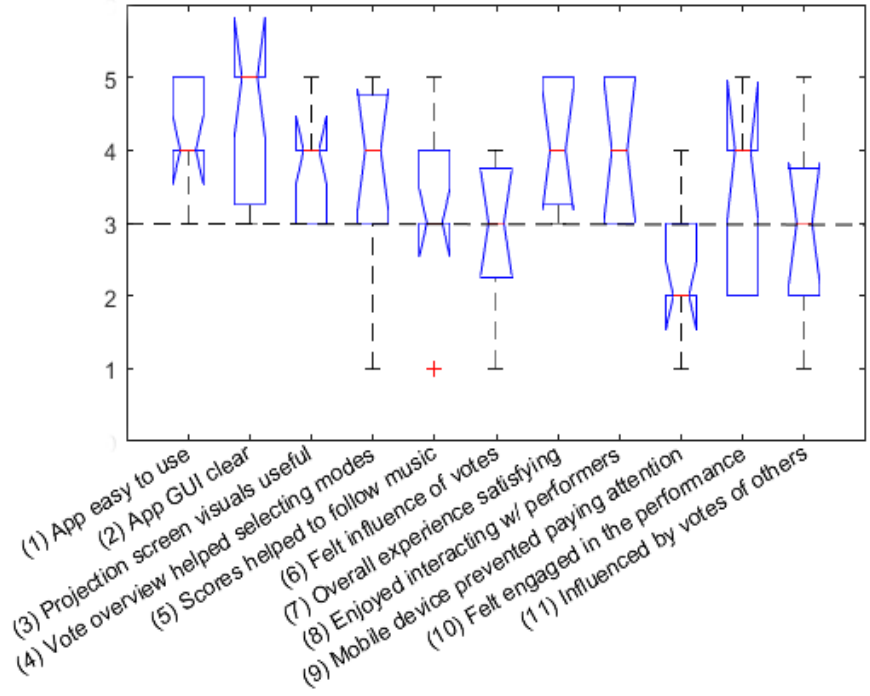

(a) Session A

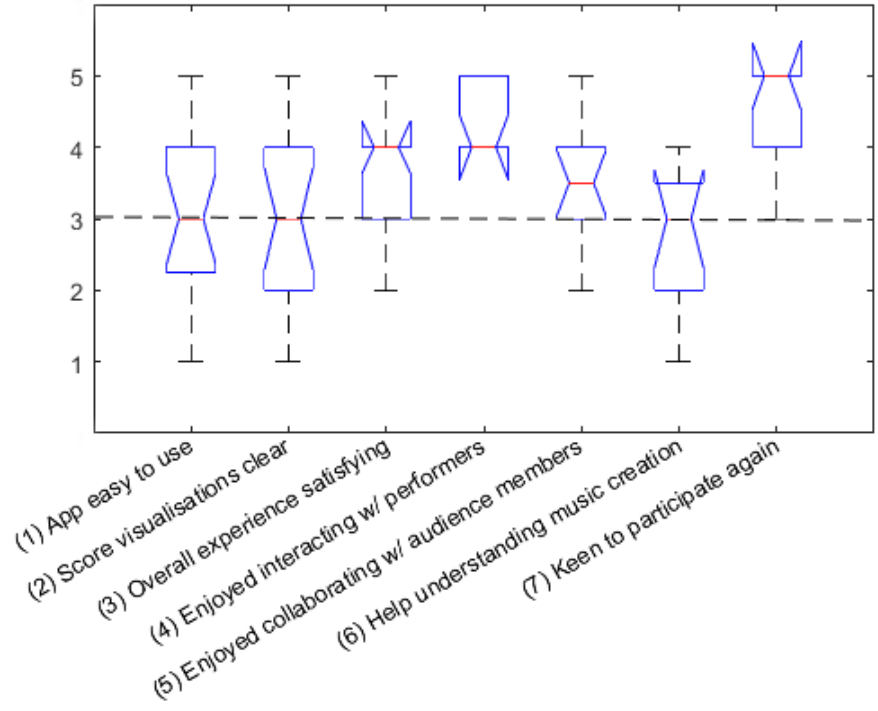

(b) Session B

Fig. 3. Box and whisker plots of audience ratings to opinion-scale questions (five-point Likert-items) for Sessions A $3 \mathrm{a}$ ) and B (3b). Notes: Question topics are reported in the labels of the $x$-axis (certain topics are common to SA and SB, whilst other differ). Notches represent the 95\% confidence interval about the median value. The format of the five-point Likert-items is as follows: 1 (Strongly disagree), 2 (Disagree), 3 (Neutral), 4 (Agree), 5 (Strongly agree). The dashed line indicates the neutral level (3). For all the topics expressing enabling factors (i.e. all the topics but one), a significant agreement to the topic highlights a positive aspect of the system. Agreement is considered significant when the lower limit of the median notch is above 3 (neutral level).

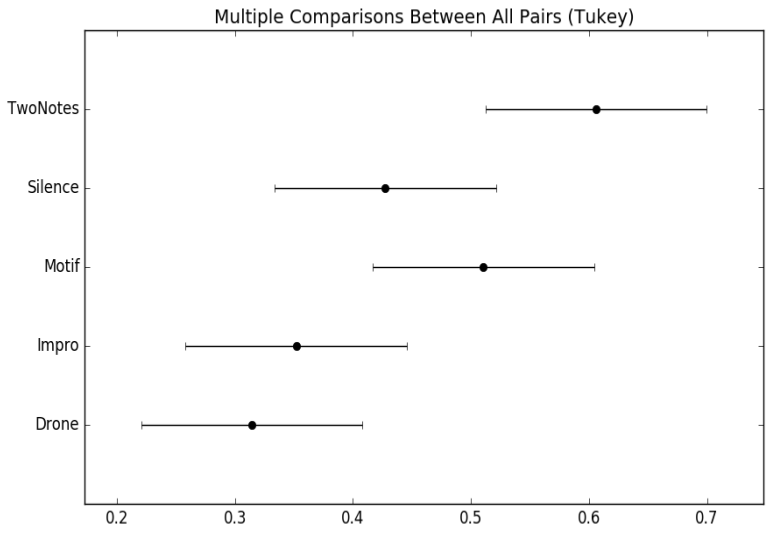

Fig. 4. Tukey multiple comparison post hoc test between participants' average playing mode vote frequency in 9 musical pieces. Note: Vote frequency (in amount per minute) and modes are reported on the $x$-and $y$ - axes, respectively.

vote frequency between sessions for each mode was similar. Results show that participants actively interacted with the system and voted on average 2.21 times per minute (sum of individual mode mean vote frequencies). The main effect of Mode was significant, $F(4,35)=7.31, p<.0005$, showing that the mean vote frequency between modes for each session differed. A Tukey multiple comparison test (see Figure 4 indicated three significantly different between-mode vote frequencies: TwoNotes vs Drone and Improvisation, and Drone vs Motif. The fact that participants significantly preferred some modes over the others suggests that the interaction was not random but guided by creative decisions. TwoNotes and Motif seem to be voted for more frequently, maybe because they are more discernible and lead to more variations than e.g. Drone.

5.4.2.2 Help interaction data: No significant differences were found in the participant average frequency of use of the app Help button between sessions, denoted $\mathrm{H}^{j}(\mathrm{k})$, $\mathrm{F}(1,13)=0.97, \mathrm{p}=0.34$. During the 14 pieces from $\mathrm{SA}$ and $\mathrm{SB}$, the Help button was used on average $\bar{H}=0.21$ times per minute per participant (once every 5 mins) which is less frequent than for any of the modes (see Figure 4 ). In SA where all participants took part in the four pieces, a linear regression model (ordinary least squares) fits the help frequency data across pieces $k$ almost perfectly, $\mathrm{H}^{1}(\mathrm{k})=0.52-0.13 \mathrm{k}$ (coefficient of determination $\mathrm{R}^{2}=.995$ ). The frequency of use of the help button decreases linearly between the first and the last pieces, no help button being invoked at all for the last piece $\left(\mathrm{H}^{1}(4)=0\right)$. This suggests that after a learning curve (corresponding to three pieces of four minutes), the participants understand how to interact with the system well.

5.4.2.3 Locus of attention: We observed from video recordings that audience members would switch their attention between the stage and their mobile devices when they interacted. Video analyses show that on average audience members spent $34 \%$ of the total duration of the concert looking at their mobile device (estimation based on five participants who could be tracked in the video). This may suggest that audience members spend half time (about $1 / 3$ of total duration) focusing on the mobile app than directly focusing on the performers (about $2 / 3$ of total duration). However, the limited number of participants who could be tracked prevent generalisation and we rely on other behavioral data such as the interaction logs presented above 
to discuss creative engagement during the performance.

\subsubsection{Thematic Analyses}

Opinion-scale questions from the survey were coupled to open-ended questions inviting participants to explain the reasons of their choice. Other open-ended questions addressed what they liked and disliked, how they positioned such interactive performances compared to traditional ones, how did they feel about "conducting" performers, which musical attributes they wished to interact with, and how they would improve the system. Inductive ('bottom-up') thematic analyses [29] of audience and performer feedback in SA and SB were conducted by the first and last authors. We present and discuss in Tables 1, 2, 3 and 4 , the main themes which emerged from the joint analysis.

\subsubsection{Modifications}

Several modifications were conducted after SA (and before SB) to improve the system. The results from the SA survey highlighted that equal vote situations hindered the clarity of visual feedback and the sense of agency as multiple modes were displayed on the graphical scores for performers to choose from (see Figure 1b. In order to minimise situations of equal votes, we replaced constant vote weights with dynamic weights $W(t)$ linearly decreasing over time (by favoring most recent votes, the sum of vote weights is unlikely to be equal across participants as votes rarely occur at the same time). For each mode $i, W_{i}(t)=\beta(T-t)$, where $\beta$ and $\mathrm{T}$ are predefined constants $(\beta=100$ and $\mathrm{T}=10 \mathrm{~s}$ were used in SB; this ensures that when sampling the function every $100 \mathrm{~ms}$ for smooths animations in the app UI vote weights remain integers which is easier to deal with in client/server transactions). Performer feedback during rehearsal in SB led us to increase the size of the mode symbols in graphical scores as one of them was color-blind. This illustrates a noteworthy benefit of participatory design as we had not accounted for this accessibility issue beforehand. An example of the modified interface combining dynamic voting and increased symbol size is provided in Figure $1 \mathrm{C}$ To make the interaction model clearer in the mobile client app and avoid game-like interactions (e.g. fast tapping), mode buttons were kept disabled for a certain threshold time $T$ after a vote $(T=10 \mathrm{~s}$ was the minimal interval between two votes in SB whilst no threshold was used in SA).

\subsubsection{Suggestions}

Several of the SB participants provided interesting suggestions which could form the basis of future developments; for instance, to replace the voting system by "giving alternate control to audience members although this wouldn't scale very well", to add playing modes (e.g. based on "mood", which the last author addresses in [7|), to remove the vote numbers from app UI (see Figure 1a) as they could be a source of distraction, to use semantic descriptions in the visuals to better explain the system, to facilitate the identification of performers using garments (" $t$-shirts with their shapes"), and to use scrolling in the graphical score.

\section{Discussion}

The subjective evaluations and behavioral and qualitative analyses provided rich data for assessing our system against the initial design requirements (see DRs in Section 3), giving us insights into how to improve it.

\subsection{Facilitating Audience Creative Participation}

The results demonstrate a significant ability of Open Symphony to support audience creative participation (DR1). In both sessions audiences enjoyed interacting with performers. A positive sense of agency and creative participation emerged from self-reports, where participants said they valued their influence on the music, the ability to interact spontaneously in a live context, and the collaboration with performers. Compared to traditional performances, the Open Symphony performances were qualified more "open", "engaging", "empowering", "unusual".

\subsection{Supporting Performers' Musical Expression}

Regarding support for performers' musical expression (DR2), the system challenged the creativity of performers, providing a novel framework for musical improvisation, structured by "necessary restrictions". Performers appreciated sharing creative roles with the audience, which they considered as the "composer" and felt they had enough space for interpretation. This indicates that the system successfully balanced the creative input between audience and performers.

\subsection{Ensuring Usability and Inclusion}

In reviewing the ability to allow responsive interactions (DR3) and to offer an intuitive system for musically untrained users (DR4), audience participants (namely in SA) did refer to the design's "simplicity" and "intuitiveness." However, prior instructions and a learning curve are required to fully understand how to negotiate the interactions and make creative decisions. Data from SA suggests that participants no longer require help after they have been adequately briefed and have used the system for three performances. Understandability issues emerged in SB when these criteria were not met, principally for non-musicians who skipped instructions and only stayed for one or two performances, without gradual introduction of modes as in SA. Relatedly, graphical score visualisations were not consistently found clear. In SA, visualisations suffered from cluttered multiple modes due to equal votes, a problem subsequently solved (see Figures $1 \mathrm{~b}$ and $1 \mathrm{c}$ ). In SB, the lack of instructions and short participation time were barriers to understanding graphical information. Although it was not one of our goals to make a fully self-explanatory system, the design could be improved to enable participants without musical expertise to rapidly infer the correct conceptual model about the system. Using semantic information in addition to visual clues, as suggested by one participant, could be a way to bridge the gap between symbols and function. Although graphical scores helped performers follow directions, they also disconnected them from the audience, forcing their locus of attention toward the screen. Disconnection from the audience was reported in SA, where the 
TABLE 1

Thematic Analysis of Audience Feedback in Session A. Note: Codes identify a feature of the data (semantic content or latent) which was judged of interest by the analyst. Themes were obtained by re-focusing the analysis at a broader level, by sorting the different codes and collating all the relevant coded data within the identified themes [29].

\begin{tabular}{l} 
Theme (no of codes) \\
\hline 1. Agency \& Creative partic- \\
ipation (13)
\end{tabular}

2. Lack of Agency (10)

3. Musical Attributes for Creative Participation (10)

4. Learnability (6)
Discussion

Several audience participants felt a positive sense of agency (e.g. "It was the direct input I had towards the performance which made me feel like I had considerable influence.") and enjoyed participating to the musical creation with performers (e.g. "I was very happy to 'play' with them.") which is in line with the ratings (see Section 5.4.1). Audience members stated that they were influenced by other members' choices, namely the majority ("I initially tried to change existing votes, or go with the group to get a clear outcome."), result in line with findings from [19]. Group interaction could also cause concerns ("Sometimes I felt a bit peer-pressurized.").

The feeling of agency could be limited due to the choice of others ("my choice does not always come first", "I didn't have direct control"), that of performers ("the performer chose what was ultimately played"), or lacking the freedom to choose which performer to interact with.

A large number of additional musical attributes for creative participation were suggested by the audience such as "key", "tempo", "rhythm", "duration", "dynamics", "octave" and audio effects like "reverb" and "delay". This indicates that audience participants would be eager to control additional sound/music dimensions. However we kept a simple five-mode interaction model to avoid cognitive overcharging and to keep the system opened to audience participants without musical knowledge.

Some of the audience participants felt that the user interface of both the mobile app and visual client were "intuitive and straightforward". However this is to be nuanced with the ratings which showed that the score visualisations were not found significantly clear. Negative feedback included a misunderstanding of the interaction mechanism with the mobile app ("I initially thought the more you tap on a symbol the more votes you submit"), which was later solved (see Section 5.4.4.

\begin{tabular}{ll}
\hline 5. Responsiveness (6) & $\begin{array}{l}\text { Latency issues emerged either due to technical reasons (e.g. "no immediate feedback of } \\
\text { the number of votes") or due to the interaction pathways and the intrinsic "time delay" } \\
\text { between votes and musicians' responses. }\end{array}$ \\
\hline 6. Engagement (5) & $\begin{array}{l}\text { In line with the ratings, several audience participants reported that the interaction made } \\
\text { them feel "engaged" in the performance, for instance because they "paid close attention } \\
\text { to the musician and how they were reacting to different input" or that their "presence } \\
\text { was not passive, but instead, very active". However some participants didn't engage } \\
\text { with the music being produced and "missed the interaction between musicians". }\end{array}$ \\
\hline 7. Distraction (3) & $\begin{array}{l}\text { Sources of distractions due to notifications from other mobile phone applications were } \\
\text { reported. Possible solutions could be to use a native app (rather than a web app) or a } \\
\text { dedicated H/W interface such as a wearable device. }\end{array}$ \\
\hline 8. Identification (3) & $\begin{array}{l}\text { Difficulty in identifying their allocated performer was reported by some audience } \\
\text { participants ("It can also be useful to know which performer you are controlling."). }\end{array}$ \\
\hline 9. Game (2) & $\begin{array}{l}\text { Two participants found the interaction to be game-like probably due to the social voting } \\
\text { and visual elements. This could also be a source of distraction from the music, inviting } \\
\text { us to attempt to minimise this effect in future iterations of the system. }\end{array}$ \\
\hline
\end{tabular}

visual display for performers was located on one side of the stage. This was improved in SB, where the visual display was placed at the front of the stage on the floor (see Figure $2 \mathrm{~b}$. Placing the visual display for performers behind the audience or using augmented reality glasses could further alleviate the sense of disconnection. Another possibility is to convey audience directions using color codes and stage lights on each of the performer. Other drawbacks of the system for audience participants were a felt lack of agency and responsiveness in some cases, indicating that DR3 was only partially met. Reasons included the voting system, which dilutes personal choices; not being able to identify the associated performer; and the limited number of musical attributes to control. The minimal allowed time interval $\mathrm{T}$ between two votes could be reduced in an attempt to increase responsiveness; however, if the interval is too small, it could give the interactions a game-like facet, which would go against the artistic intent. We could investigate voting systems other than plurality, but there are currently no optimal solutions for groups. We could also consider providing audience participants with full control, but over different musical attributes (shared roles in composition), assuming complexity and scalability can be factored in. 
TABLE 2

Thematic Analysis of Performer Feedback in Session A

\begin{tabular}{|c|c|}
\hline Theme (no of codes) & Discussion \\
\hline 1. Visualisation (9) & $\begin{array}{l}\text { The graphical scores proved useful in supporting music making decision ("it was pretty } \\
\text { clear where we were in the music", "It gave something to follow and to understand } \\
\text { the duration of the piece."). However, other performers' scores and the overview of } \\
\text { audience choices were not necessary for some ("I was listening to what others were } \\
\text { doing, and at times looking, but I was mostly focused on my own.") and could be } \\
\text { sources of distraction for others ("it distracts one from listening to the other players"). } \\
\text { For one performer, another drawback of using a screen was that it disconnected them } \\
\text { from the audience ("As a performer I felt little relation to the audience since I was } \\
\text { focused on a screen."). }\end{array}$ \\
\hline 2. Creativity (4) & $\begin{array}{l}\text { The system pushed performers to explore their creativity in novel ways ("Gave me } \\
\text { options to create in a different way to how I normally do", "freedom to experiment with } \\
\text { notes and styles"). Compared to free improvisations, the system provided them with a } \\
\text { "structure" and "restrictions" deemed to be "necessary" by some. }\end{array}$ \\
\hline $\begin{array}{l}\text { 3. Musical Attributes for } \\
\text { Creative Participation (4) }\end{array}$ & $\begin{array}{l}\text { Similarly to audience members, all the performers suggested additional music param- } \\
\text { eters or styles to play (i.e. "Baroque and improv etc", "dynamics or tempo", "keys, } \\
\text { moods"). }\end{array}$ \\
\hline 4. Participatory creation (3) & $\begin{array}{l}\text { Performers valued sharing the control over music production with the audience ("puts } \\
\text { the musician and the composers (audience) on equal ground"). This proved successful } \\
\text { because the artistic structure left them some space for interpretation ("It was nice to be } \\
\text { able to make the decision based on your ears rather than what was dictated."). }\end{array}$ \\
\hline $\begin{array}{l}\text { 5. Lack of Compositional } \\
\text { Structure (2) }\end{array}$ & $\begin{array}{l}\text { Two performers found that the music lacked of compositional structure ("There is not } \\
\text { an overall 'compositional' structure - musicians respond to what is directed at the time } \\
\text { rather than what has been before.", "I liked the } 2 \text { modes stuff, but with } 5 \text { modes in play } \\
\text { things quickly became chaotic."). We partly attributed such impression of "chaos" for } \\
\text { five modes in SA to equal vote situations leading to multiple modes at the same time } \\
\text { (see Figure 1b), and made modifications to prevent this (see Section 5.4.4. }\end{array}$ \\
\hline
\end{tabular}

\subsection{Facilitating Audience Engagement}

Enabling agency and participation fostered audience engagement in the performance (DR5), as participants paid close attention to performers and felt active. Creative voting strategies were diverse, demonstrating system appropriation based on personal preference; some favored certain playing modes, some based their decisions only on the music, and others were influenced by multiple modalities (the visualisations, others' votes, and the music). In SB, a strong theme of pleasure and enjoyment emerged. Participants had positive affective responses for artistic reasons (such as the quality of the music, the skills, and the performers' dedication), intellectual reasons (such as the cognitive tasks of voting and evaluating feedback), and also nonmusical reasons (the stage setting and visual display with live graphical scores enriching the performance experience), an aspect also observed in |30|.

\subsection{Supporting Musical Co-Composition}

In terms of support for musical co-composition (DR6), some participants thought the music was "excellent" and "unusual". However, other participants did not engage with it, which might be due to a lack of "overall compositional structure" (as reported in SA), a lack of "modulation" (as reported in SB), or a genuine dislike of experimental music. The musical component should be developed further to ensure that audience compositional directions can form a coherent whole, yet integrating layers of complexity (SB).
We could introduce a system of meta-rules to provide a curated, overall direction for Open Symphony interactive pieces, while keeping internal parts responsive and mutable.

\subsection{Ensuring Scalability}

Similar levels of interaction (vote frequency) were found between sessions composed of performances with varying number of participants (see Section 5.4.2). However, these comparisons were conducted between small- to mediumscale audiences (with 10-30 interacting participants). Scalability (DR7) tests including larger audiences should be conducted to test the impact of audience size, using both attitudinal and behavioral measures.

\section{CONCLUSION AND FUtURE WORK}

We presented Open Symphony, a novel system for participatory live music performance involving audience-performer interaction using web technology and data visualisation. The system was the result of an iterative participatory design approach involving a team of researchers, designers, artistic director and performers, thus covering multidisciplinary skills. The system transforms the traditional unidirectional musical chain by adding creative communication in the reverse direction, from the audience to performers. Our design favored simple affordances (voting for playing modes) and multimodal interaction mixing musical and visual elements. Results from two evaluations which involved 
TABLE 3

Thematic Analysis of Audience Feedback in Session B

\begin{tabular}{|c|c|}
\hline Theme (no of codes) & Discussion \\
\hline 1. Learnability (26) & $\begin{array}{l}12 \text { out of } 20 \text { participants (11 musicians, one non musician) did not report understand- } \\
\text { ability issues. However eight participants (six non-musicians, two musicians) expressed } \\
\text { difficulties to understand e.g. the meaning of the symbols, the effect of the votes, the } \\
\text { graphical scores, and expressed the need for explanations (it was "confusing at first" } \\
\text { but "once briefed it was fine"). Contrary to SA, not all participants from SB attended the } \\
\text { introduction as they could join at any point. Participants from SB also took part in } 1.4 \\
\text { performances on average compared to four for SA. This shows that an introduction } \\
\text { is required for audience participants especially for non-musicians, and a minimum } \\
\text { number of performances is needed to support the learning curve. }\end{array}$ \\
\hline
\end{tabular}

2. Pleasure/Enjoyment (19) Many participants communicated their enjoyment of the experience. Reasons were varied, from intellectual pleasure ("This simply rocked and was very fun but also very cerebrally satisfying.") to emotional contagion seeing skilled performers enjoying interacting together, and the quality of the music ("music was excellent").

3. Design (18) Many positive comments were made regarding the design of the interfaces ("I liked the simplicity of the concept, the polish of the app and main screen.", "not cluttered") and the feedback provided ("I could easily select what I wanted and see total votes for a certain playing mode"). Other comments contrasted the aesthetics versus the clarity ("didn't know what symbols meant [...] but very pretty").

\begin{tabular}{ll}
\hline $\begin{array}{l}\text { 4. Lack of Agency/Control } \\
\text { (12) }\end{array}$ & $\begin{array}{l}\text { As in SA, several factors hindering the sense of agency were identified; e.g. the voting } \\
\text { system causing individual decisions to get "diluted" ("I could not see my personal } \\
\text { influence") and the lack of feedback or misunderstanding of the system ("Wasn't sure if } \\
\text { my input reached the performer."). }\end{array}$ \\
\hline 5. Interactivity (8) & $\begin{array}{l}\text { The interactivity of the system was a source of positive emotional experience ("Thought } \\
\text { it was lots of fun and interactive with the audience and musicians"). The possibility of } \\
\text { "interacting with LIVE performance" was the aspect some liked the most. }\end{array}$ \\
\hline 6. Interest/Curiosity (7) & $\begin{array}{l}\text { Several participants found the concept "interesting" and were curious to see future } \\
\text { developments. }\end{array}$ \\
\hline 7. Voting strategy (7) & $\begin{array}{l}\text { Voting strategies were diverse such as "personal preference", multimodal considerations } \\
\text { taking into account the visualisation, the votes and the music, trial and error and } \\
\text { randomness, the music dynamics, and the votes from others. }\end{array}$ \\
\hline 8. Responsiveness (5) & $\begin{array}{l}\text { As in SA, issues of responsiveness were reported (e.g. "not responsive enough for } \\
\text { effective music", "a sense of asynchrony between votes and performer execution of } \\
\text { vote"). }\end{array}$ \\
\hline 9. Originality/Spontaneity & $\begin{array}{l}\text { Interestingly, several participants felt attracted by how "unconventional" the music was } \\
\text { and that its "spontaneity" created something new. }\end{array}$ \\
\hline 10. Agency/Engagement (4) & $\begin{array}{l}\text { As in SA, the sense of agency towards the music was valued positively ("you feel like } \\
\text { the music is influenced by you") also respecting the place of the musicians ("i liked } \\
\text { that i could influence the music but musicians could choose what to do without my } \\
\text { feedback"). }\end{array}$ \\
\hline 11. Identification (3) & $\begin{array}{l}\text { Similarly to SA, a few participants were unsure about which performer they were } \\
\text { controlling. }\end{array}$ \\
\hline 12. Technical issues (3) & $\begin{array}{l}\text { A small number of technical issues occurred either due to type of browser/mobile phone } \\
\text { and the network which was at times cluttered. }\end{array}$ \\
\hline 13. Augmented stage (3) & $\begin{array}{l}\text { Several participants reported to have liked the performance setting due to the way the } \\
\text { stage was presented or the "visual display". }\end{array}$ \\
\hline
\end{tabular}

about 120 interacting participants ( 35 completing surveys) showed that audience and performer participants valued sharing the process of musical creation. Both groups of users felt challenged by the novelty, audience members who took creative decisions and decoded their effects, performers, who followed scores generated while they were playing. Such challenges lead to positive affective responses for some audience participants who felt engaged in the performance and closely connected to performers, and to some frustra- tion for others who misunderstood the system or wished to have more control. Open Symphony showed the potential to create performances that are "open", "engaging", "empowering" and "unusual". Future analyses will investigate participant creative interactions using time series modeling. We are also interested in exploring applications for music pedagogy and audience engagement agendas. 
TABLE 4

Thematic Analysis of Performer Feedback (Two) in Session B

\begin{tabular}{|c|c|}
\hline Theme (no of codes) & Discussion \\
\hline 1. Usability / Design (7) & $\begin{array}{l}\text { The system was judged easy to learn and to use by the two performers ("I thought } \\
\text { the digital elements were easy to follow and and intuitive.", "Everything made sense, } \\
\text { including the way the rehearsals went and the way we performed."), only requiring } \\
\text { little preparation before live performance ("I don't think there has to be much rehearsal. } \\
\text { It seems self-explanatory."). The graphic design was also judged to be clear and aesthetic } \\
\text { ("I loved the presentation! The symbols were beautiful and the layout was very very } \\
\text { clear."). }\end{array}$ \\
\hline 2. Musical complexity (4) & $\begin{array}{l}\text { The two performers expressed that the musical complexity could be increased, e.g. } \\
\text { through modulation ("I would be interested in how you can maybe shift tonalities/and } \\
\text { or modes within the piece to add another layer of complexity and color to the } \\
\text { improvisation.") or through harmony/layering ("I think the composition could even } \\
\text { be a little more complex! Maybe a few different chords or different layers."). }\end{array}$ \\
\hline 3. Satisfaction (4) & $\begin{array}{l}\text { The two performers were very positive about their experience from the rehearsal to the } \\
\text { live execution (e.g. "All in all, great experience."). }\end{array}$ \\
\hline $\begin{array}{l}\text { 4. Audience engagement / } \\
\text { Pedagogical outreach (2) }\end{array}$ & $\begin{array}{l}\text { The potential of the system for audience engagement and music pedagogy was fleshed } \\
\text { out by one of the performers ("I think, expanded, this could be a really interesting way } \\
\text { to engage non-musicians and help them become familiarized with classical music."). }\end{array}$ \\
\hline
\end{tabular}

\section{ACKNOWLEDGMENTS}

We thank the project music director, Kate Hayes, and the performers from Guildhall School of Music \& Drama and the University of California, Berkeley. This work is partly supported by the FAST-IMPACt EPSRC project (EP/L019981/1), the Centre for Digital Music EPSRC Platform Grant (EP/E045235/1), the EU H2020 Audio Commons project (688382), QMUL's Centre for Public Engagement, the China Scholarship Council, and Arts Council England (Sound and Music Organisation Audience Labs).

\section{REFERENCES}

[1] T. Turino, Music as Social Life: The Politics Of Participation. University of Chicago Press, 2008.

[2] J. Radbourne, K. Johanson, H. Glow, and T. White, "The audience experience: Measuring quality in the performing arts," International Journal of Arts Management, pp. 16-29, 2009.

[3] E. Lindström, P. N. Juslin, R. Bresin, and A. Williamon, “'Expressivity comes from within your soul': A questionnaire study of music students' perspectives on expressivity," Research Studies in Music Education, vol. 20, pp. 23-47, 2003.

[4] J. Vies, R. Clarke, P. Wright, J. McCarthy, and P. Olivier, "Configuring participation: on how we involve people in design," in Proc. of CHI, 2013, pp. 429-438.

[5] E. Gómez, M. Grachten, A. Hanjalic, J. Janer, S. Jordà, C. F. Julià, C. Liem, A. Martorell, M. Schedl, and G. Widmer, "PHENICX: Performances as highly enriched and interactive concert experiences," in Proc. of Stockholm Music Acoustics Conference (SMAC), 2013. [Online]. Available: http://citeseerx.ist. psu.edu/viewdoc/summary?doi=10.1.1.352.5250

[6] J. Freeman, "Large audience participation, technology, and orchestral performance," in Proc. of the International Computer Music Conference, 2005, pp. 757-760.

[7] G. Fazekas, M. Barthet, and M. B. Sandler, "The Mood Conductor system: Audience and performer interaction using mobile technology and emotion cues," in Proc. of the Int. Symposium on Computer Music Multidisciplinary Research (CMMR), 2013. [Online]. Available: http://www.cmmr2013.cnrs-mrs.fr/ Docs/CMMR2013Proceedings.pdt

[8] J.-P. Lambert, S. Robaszkiewicz, and N. Schnell, "Synchronisation for distributed audio rendering over heterogeneous devices, in html5," in Proc. of Web Audio Conference (WAC), Atlanta, USA, 2016. [Online]. Available: http://hdl.handle.net/1853/54598
[9] A. Clément, F. Ribeiro, R. Rodrigues, and R. Penha, "Bridging the gap between performers and the audience using networked smartphones: the a.bel system," in Proc. of Int. Conf. on Live Interfaces, 2016.

[10] S. Kattwinkel, Audience participation: Essays on inclusion in performance. Greenwood Publishing Group, 2003, no. 101.

[11] M. Barthet, F. Thalmann, G. Fazekas, M. B. Sandler, and G. A. Wiggins, "Crossroads: Interactive music systems transforming performance, production and listening," in Proc. of CHI Music and HCI Workshop, 2016. [Online]. Available: http: //qmro.qmul.ac.uk/xmlui/handle/123456789/12502

[12] D. Maynes-Aminzade, R. Pausch, and S. Seitz, "Techniques for interactive audience participation," in Proceedings of the 4th IEEE International Conference on Multimodal Interfaces. IEEE Computer Society, 2002, p. 15. [Online]. Available: http://dl.acm.org/citation.cfm?id=847725

[13] D. Mazzanti, V. Zappi, D. Caldwell, and A. Brogni, “Augmented stage for participatory performances," in Proc. of NIME, 2014.

[14] K. Hayes, M. Barthet, Y. Wu, L. Zhang, and N. Bryan-Kinns, "A participatory live music performance with the Open Symphony system," in Proceedings of the 2016 CHI Conference Extended Abstracts on Human Factors in Computing Systems, 2016, pp. 313316. [Online]. Available: http://doi.acm.org/10.1145/2851581. 2889471

[15] T. Cerratto-Pargman, C. Rossitto, and L. Barkhuus, "Understanding audience participation in an interactive theater performance," in Proc. of the 8th Nordic Conference on Human-Computer Interaction: Fun, Fast, Foundational. ACM, 2014, pp. 608-617.

[16] J. Schwarz, D. Klionsky, C. Harrison, P. Dietz, and A. Wilson, "Phone as a pixel: Enabling ad-hoc, large-scale displays using mobile devices," in In Proc. of CHI, 2012, pp. 2235-2238.

[17] M. Barthet, G. Fazekas, A. Allik, F. Thalmann, and M. B. Sandler "From interactive to adaptive mood-based music listening experiences in social or personal contexts," J. Audio Engineering Society, vol. 64, no. 9, pp. 673-682, 2016. [Online]. Available: http://www.aes.org/e-lib/browse.cfm?elib=18376

[18] F. Berthaut, D. Martinez Plasencia, M. Hachet, and S. Subramanian, "Reflets: Combining and revealing spaces for musical performances," in In Proc. of NIME, 2015.

[19] M. Feldmeier and J. A. Paradiso, "An interactive music environment for large groups with giveaway wireless motion sensors," Computer Music Journal, vol. 31, no. 1, pp. 50-67, 2007.

[20] L. Zhang, Y. Wu, and M. Barthet, "A web application for audience participation in live music performance: The Open Symphony use case," in Proc. of International Conference on New Interfaces for Musical Expression (NIME), 2016.

[21] N. Weitzner, J. Freeman, S. Garrett, and Y.-1. Chen, "massMobile - 
an Audience Participation Framework," in Proc. of the International Conference on New Interfaces for Musical Expression, 2012, pp. 92-95.

[22] S. Jordà, "On stage: the Reactable and other musical tangibles go real," Int. J. Arts and Technology, vol. 1, no. 3/4, pp. 268-287, 2008.

[23] B. Morris. (2015) Conduct. [Online]. Available: http://www. conduction.us/

[24] M. Barthet, P. Depalle, R. Kronland-Martinet, and S. Ystad, "Acoustical correlates of timbre and expressiveness in clarinet performance," Music Perception, vol. 28, no. 2, pp. 135-153, 2010.

[25] C. Ware, Information visualization: perception for design. Elsevier, 2012.

[26] Y. S. Park and S. H. Han, "Touch key design for one-handed thumb interaction with a mobile phone: Effects of touch key size and touch key location," International Journal of Industrial Ergonomics, vol. 40, no. 1, pp. 68-76, 2010.

[27] T. Sauer, Notations 21. Mark Batty Pub, 2009.

[28] K. A. Hallgren, "Computing inter-rater reliability for observational data: an overview and tutorial," Tutorials in quantitative methods for psychology, vol. 8, no. 1, p. 23, 2012.

[29] V. Braun and V. Clarke, "Using thematic analysis in psychology," Qualitative research in psychology, vol. 3, no. 2, pp. 77-101, 2006.

[30] S. Thompson, "Audience responses to a live orchestral concert," Musicae Scientiae, vol. X, no. 2, pp. 215-244, 2006.

Yongmeng $\mathbf{W u}$ is a $\mathrm{PhD}$ student in the Media and Arts Technology Centre for Doctoral Training, Queen Mary University of London. Her research interests include human-computer interaction, user experience, evaluation of interaction design (aesthetic experience, creativity, and engagement), and support of non-musicians' creative engagement with novel musical interfaces. Wu has an MA in design (human computer interaction) from Hunan University, China. Contact her at yongmeng.wu@qmul.ac.uk.

Leshao Zhang is a PhD student in the Media and Arts Technology Centre for Doctoral Training, Queen Mary University of London, and a member of the Cognitive Science Research Group. His research interests include human-computer interaction and virtual reality. Zhang has an MSc in media and arts technology from Queen Mary University of London. Contact him at leshao.zhang@qmul.ac.uk.

Nick Bryan-Kinns is a reader in interaction design at Queen Mary University of London, and director of the EPSRC+AHRC Media and Arts Technology Centre for Doctoral Training. His research interests include the evaluation of interaction design, focusing on creativity, mutual engagement, and audio. Bryan-Kinns has a PhD in human-computer interaction. He is a Fellow of the British Computer Society. Contact him at n.bryan-kinns@qmul.ac.uk.

Mathieu Barthet Barthet is a lecturer and technical director of the Media and Arts Technology Studios at Queen Mary University of London. His research lies at the intersections of music informatics, human-computer interaction, affective computing, and perception. Barthet has a PhD on musical timbre analysis/synthesis from Aix-Marseille University and Centre National de Recherche Scientifique's Laboratory of Mechanics and Acoustics. He is a member of IEEE, the Association for Computing Machinery, and the Audio Engineering Society. Contact him at m.barthet@qmul.ac.uk. 